\title{
Postpartum Depression: Prevalence and Associated Risk Factors Among Women in Sindh, Pakistan
}

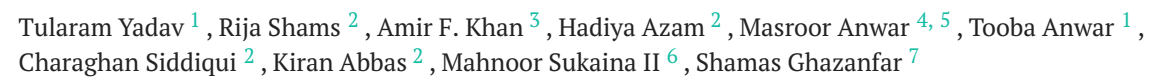

1. Internal Medicine, Jinnah Sindh Medical University, Karachi, PAK 2. Internal Medicine, Jinnah Postgraduate Medical Centre, Karachi, PAK 3. General Surgery, Lady Reading Hospital MTI, Peshawar, PAK 4. Internal Medicine, Hayatabad Medical Complex, Peshawar, PAK 5. Surgery, Lady Reading Hospital MTI, Peshawar, PAK 6. Internal Medicine, Karachi Medical and Dental College, Karachi, PAK 7. Internal Medicine, Dow University of Health Sciences, Karachi, PAK

Corresponding author: Kiran Abbas, kiranabbas2020@gmail.com

\section{Abstract}

\section{Introduction}

Postpartum depression (PPD) is defined as the onset of depressive symptoms within six weeks of childbirth. PPD is more common in resource-constrained countries as compared to developed countries. The study aimed to evaluate the factors associated with PPD among women in Sindh, Pakistan.

\section{Methods}

A multi-centre, cross-sectional study was conducted at three major tertiary care setups in Sindh, Pakistan. All women presenting to the outpatient department within six weeks of giving live birth were eligible to participate. All women who had stillbirths, abortions, or were treated for a past psychiatric illness or neurological disease were excluded from the study. The Edinburgh postpartum depression scale (EPDS) was used as a screening tool. All socio-demographic factors were documented in a predefined pro forma. The data was analyzed using Statistical Package for Social Sciences (SPSS, Version 26, IBM, Chicago, IL).

\section{Results}

According to the Edinburgh postpartum depression scale (EPDS), the incidence of postpartum depression in the current study population was $19.3 \%$. Of these, 12 (3.3\%) women had persistently thought about selfharming. Over 100 women did not receive any formal education, constituting the majority of the study population. Formula milk feeding of the newborn was significantly associated with an increased frequency of postpartum depression $(\mathrm{p}=0.0001)$.

\section{Conclusion}

Review began 12/09/2020 Review ended 12/21/2020 Published 12/22/2020

\section{() Copyright 2020}

Yadav et al. This is an open access article distributed under the terms of the Creative Commons Attribution License CC-BY 4.0., which permits unrestricted use, distribution, and reproduction in any medium, provided the original author and source are credited.
The current study highlights the significant burden of postpartum depression in Pakistan. However, the present study failed to find any significant risk factors associated with postpartum depression. Only formula milk feeding was significantly associated with a higher frequency of PPD among study patients.

Categories: Psychiatry, Psychology, Epidemiology/Public Health

Keywords: postpartum depression, breastfeeding woman, formula milk, international classification of diseases - 11 (icd-11), postpartum mental health, peripartum psychosis

\section{Introduction}

According to the International Classification Disease (ICD-10), postpartum depression (PPD) is defined as the onset of depressive symptoms within six weeks of childbirth [1,2]. Postpartum depression can manifest in new mothers as feelings of hopelessness, fatigue, unjustified guilt, and shame [3]. Sleep irregularities and reduced appetite in addition to severe mood changes are common symptoms of postpartum depression [4].

Globally, postpartum depression can affect up to $15 \%$ of mothers annually. PPD is more common in resource-constrained countries as compared to developed countries [5]. The prevalence of postpartum depression in Pakistan ranges from 28-63\% [5,6]. Certain factors have been associated with an increased risk of postpartum depression, including a history of psychiatric illness, psychological disturbance during gestation, domestic violence or poor marital relationship, and inadequate social support [7]. Low social status and lack of access to healthcare facilities are also essential factors in determining maternal and fetal outcomes $[7,8]$.

Mothers who suffer from postpartum depression are unable to take care of themselves and provide adequate 
care to the infant. This results in a non-conducive environment for the personal development of the mothers and their newborn babies. It significantly disrupts infant-mother bonding, leading to child neglect, emotional or physical child abuse [9]. Previous studies have suggested that mothers with untreated postpartum depression suffer from weight problems, substance abuse, domestic problems, and breastfeeding issues later in life $[9,10]$.

Published data are scarce on the risk factors associated with increased risk of PPD in mothers in Pakistan. Therefore, the current study was conducted to evaluate the factors in predicting PPD among women giving birth at a tertiary care center in Sindh, Pakistan.

\section{Materials And Methods}

A multi-centre cross-sectional study was conducted at three major tertiary care setups in Sindh, Pakistan, which cater to approximately one million patients, annually. The data was collected from the Jinnah Postgraduate Medical Centre (JPMC), Dr Ruth K. M. Pfau, Civil Hospital, and Abbasi Shaheed Hospital. The study was started after obtaining ethical approval from the Institutional Review Board (IRB), reference \#JSMU/IRB/2019/-167. A simple random sample technique was used to enrol participants.

All women presenting to the outpatient department within six weeks of giving live birth were eligible to participate. All women who had stillbirths, abortions, or were treated for a past psychiatric illness or neurological disease were excluded from the study. The sample size was calculated via OpenEpi sample size calculator, using a 95\% confidence interval (CI), 5.18\% margin of error (d), and $48.6 \%$ as the prevalence of postpartum depression [10]. A total sample size of 357 was determined. All patients signed informed written consent to participate in the study.

The Edinburgh postpartum depression scale (EPDS) was used as a screening tool. All socio-demographic factors were documented in a predefined pro forma. The EPDS is a self-reported questionnaire with 10 items. It assesses anhedonia, feelings of self-blame, anxiety, fear or panic, inability to cope, and thoughts of self-harm in patients [11]. Due to the participant's limited English language, the questionnaires were translated into Sindhi, Pashto, and Urdu languages. Native persons critically revised the translated questionnaires to remove any inconsistencies. The questionnaires were then administered to women. Those who could not read or write, the questionnaires were read to them, and their answers were recorded by the authors.

The data was analyzed using Statistical Package for Social Sciences (SPSS, Version 26, IBM, Chicago, IL). All continuous variables were presented as mean and standard deviation while for all categorical variables, frequency and percentage were used. The dependent variable was the occurrence of postpartum depression, and the socio-demographic variables were tagged as independent variables. Chi-square tests and independent $\mathrm{t}$-tests were used to check for the association between dependent and independent variables. A p-value of less than or equal to 0.05 was considered as significant.

\section{Results}

A total of 357 participants were enrolled in the study. The mean age (standard deviation) was 26.18 (5.53) years and ranged between 14 to 50 years. Three hundred forty-six (96.9\%) women were Muslim while only a minority were non-muslims. Over 100 women did not receive any formal education, constituting the majority of the study population (table 1). Out of the 69 women who had postpartum depression, nine (13.1\%) had a substance abuse problem. The majority were addicted to areca nuts. 


\section{Cureus}

Characteristics

N (\%)

Religion

Islam

Hinduism

Christianity

Education Status

Madrasah

Primary Class

Secondary Class

Higher Secondary Class

Bachelors

No formal education

Occupational Status

Employed

Unemployed/Homemakers
$27(7.6 \%)$

$346(96.9 \%)$

$6(1.7 \%)$

5 (1.4\%)

91 (25.5\%)

$83(23.2 \%)$

26 (7.3\%)

$29(8.1 \%)$

$101(28.3 \%)$

$17(4.8 \%)$

$340(95.2 \%)$

TABLE 1: Sociodemographic Characteristics of Study Participants

According to the Edinburgh postpartum depression scale (EPDS), the incidence of postpartum depression in the current study population was $19.3 \%$. Of these, 12 (3.3\%) women had persistently thought about selfharming indicating suicidal ideation (Table 2).

\section{Characteristics}

I have been able to laugh and see the funny side of things:

As much as always I could

Not quite as much now

Definitely not so much now

Not at all

I have looked forward with enjoyment to things:

As much as I ever did

Rather less than I used to

Definitely less than I used to

Hardly at all

I have blamed myself unnecessarily when things went wrong:

Yes, most of the time

Yes, sometimes

Not very often

No, never

I have been anxious or worried for no good reason:

No, not at all
$91(25.5 \%)$

$42(11.8 \%)$

$31(8.7 \%)$

$193(54.1 \%)$

N (\%)

$256(71.7 \%)$

$74(20.7 \%)$

$15(4.2 \%)$

$12(3.4 \%)$

$284(79.6 \%)$

$51(14.3 \%)$

$12(3.4 \%)$

$10(2.8 \%)$

245 (68.6\%) 


\section{Cureus}

\begin{tabular}{|c|c|}
\hline Hardly ever & $18(5.0 \%)$ \\
\hline Yes, sometimes & $63(17.6 \%)$ \\
\hline Yes, very often & $31(8.7 \%)$ \\
\hline \multicolumn{2}{|c|}{ I have felt scared or panicky for no very good reason: } \\
\hline Yes, quite a lot & $21(5.9 \%)$ \\
\hline Yes, sometimes & $57(16.0 \%)$ \\
\hline No, not much & $37(10.4 \%)$ \\
\hline No, not at all & $242(67.8 \%)$ \\
\hline \multicolumn{2}{|c|}{ Things have been getting on top of me: } \\
\hline Yes, most of the time & $34(9.5 \%)$ \\
\hline Yes, sometimes & $69(19.3 \%)$ \\
\hline No, most of the time & $69(19.3 \%)$ \\
\hline No, I have been coping as usual & $185(51.8 \%)$ \\
\hline \multicolumn{2}{|c|}{ I have been so unhappy that I have had difficulty sleeping: } \\
\hline Yes, most of the time & $23(6.4 \%)$ \\
\hline Yes, quite often & $44(12.3 \%)$ \\
\hline Not very often & $43(12.0 \%)$ \\
\hline Not at all & $247(69.2 \%)$ \\
\hline \multicolumn{2}{|l|}{ I have felt sad or miserable: } \\
\hline Yes, most of the time & $10(2.8 \%)$ \\
\hline Yes, Quite often & $23(6.4 \%)$ \\
\hline Not very often & $44(12.3 \%)$ \\
\hline Not at all & $280(78.4 \%)$ \\
\hline \multicolumn{2}{|c|}{ I have been so unhappy that I have been crying: } \\
\hline Yes, most of the time & $23(6.4 \%)$ \\
\hline Yes, quite often & $20(5.6 \%)$ \\
\hline Only occasionally & $34(9.5 \%)$ \\
\hline No, never & $280(78.4 \%)$ \\
\hline \multicolumn{2}{|c|}{ The thought of harming myself has occurred to me: } \\
\hline Yes, quite often & $3(0.8 \%)$ \\
\hline Yes, sometimes & $9(2.5 \%)$ \\
\hline Hardly ever & $11(3.1 \%)$ \\
\hline No, never & 334 (93.6\%) \\
\hline
\end{tabular}

TABLE 2: Study Participants' Response to the Edinburgh Postpartum Depression Scale 


\section{Cureus}

\begin{tabular}{|c|c|c|c|}
\hline \multirow{2}{*}{ Characteristics } & \multicolumn{2}{|l|}{ EPDS Score } & \multirow{2}{*}{ P-value } \\
\hline & Score $\geq 10$ & Score $<10$ & \\
\hline \multicolumn{3}{|l|}{ Educational Status } & \multirow{4}{*}{0.213} \\
\hline No formal education & $23(20.3 \%)$ & $87(30.2 \%)$ & \\
\hline Madrasah or up to class $12^{\text {th }}$ & $51(65.2 \%)$ & $176(61.1 \%)$ & \\
\hline Bachelors & $4(5.8 \%)$ & $25(8.7 \%)$ & \\
\hline \multicolumn{3}{|l|}{ Gender of the Newborn } & \multirow{3}{*}{0.595} \\
\hline Female & $29(42 \%)$ & 104(36.1\%) & \\
\hline Male & $40(58 \%)$ & $183(63.5 \%)$ & \\
\hline \multicolumn{3}{|l|}{ Feeding Method } & \multirow{3}{*}{0.0001} \\
\hline Exclusive Breast Feeding & $31(44.9 \%)$ & $191(66.3 \%)$ & \\
\hline Formula Milk Feeding & $38(55.1 \%)$ & $97(33.7 \%)$ & \\
\hline \multicolumn{3}{|l|}{ Family Type } & \multirow{3}{*}{0.461} \\
\hline Nuclear & $23(33.3 \%)$ & $83(28.8 \%)$ & \\
\hline Extended & $46(66.7 \%)$ & $205(71.2 \%)$ & \\
\hline
\end{tabular}

TABLE 3: The Sociodemographic Factors Associated with Postpartum Depression

\section{Discussion}

We reported an incidence of PPD of $19.3 \%$ in our study. Previous studies have shown a PPD prevalence somewhere between 3-69.65\%. The varying degrees of prevalence indicate that there are some sociodemographic variables at play here. In developed countries including Singapore, Netherlands, and Switzerland, the prevalence rate of PPD was $3 \%, 8 \%$, and $11 \%$ respectively [12]. However, in less developed and poor constrained countries like Pakistan, India, and Nepal, the incidence of the disease is much higher [3,12-13]. This highlights the significance of social status or resource availability and the risk of PPD in women after delivery. In contrast, the prevalence of PPD was considerably higher in Iran, Chile, South Africa, and Turkey. The reasons for this disparity in different geographic regions is unclear.

In the current study, we reported a significant association between the educational status of women and the frequency of PPD. PPD was more prevalent in women with poor educational status compared to those with higher education status. In a study by Khan et al., similar conclusions were culminated [14]. Similarly, in a study from Japan, over 90,000 women were evaluated to find an association between education status and risk of developing PPD. The study revealed that higher education status among women was associated with a decreased rate of PPD [15]. The education level of a woman is frequently used to assess the socioeconomic status indirectly. Individuals with lower socioeconomic status have a higher risk of developing a myriad of psychiatric illnesses. The current study reported that PPD was more prevalent in women with lower socioeconomic status highlighting it as an independent risk factor for postpartum depression.

The present study also found an association between women who were exclusively breastfeeding and a decreased rate of postpartum depression compared to women providing formula milk to their newborns. Previously, a study by Shah et al. reported a significant relationship between the incidence of PPD and exclusive breastfeeding [12]. Ystrom in 2012, revealed that women with postpartum depression or anxiety were a highly vulnerable population. In support of the current findings, these women were likely to cease breastfeeding their newborns after which their symptoms related to depression and anxiety worsened substantially [16].

Our study also found a link between the joint family system and postpartum depression. Women more frequently suffered from PPD if they lived in a joint family system compared to women who resided in a nuclear family system. Naveed and Naz [17] drew the same conclusions about the correlation between the joint family system and increased risk of PPD. Social support is significant for the well-being of new mothers. Essentially, women who did not receive any social support were more prone to suffering from PPD [18]. In a study by Kim et al., it was found that women irrespective of their age had five times the risk of developing postpartum depression if no social support was provided to them after delivery [19]. 
Our study was not without limitations. Firstly, during data collection, the authors and the participants faced language barriers since many of the women belonged to rural areas and could not speak or write the Urdu language fluently. To minimize the language barrier, the authors had the questionnaire translated into several local languages which were then proofread by native persons. However, many women did not have any formal education and were unable to read or write. For such cases, the authors narrated the questionnaires to the participant who then answered accordingly. Secondly, the study was cross-sectional and did not keep a follow-up track of patients. Further studies with longitudinal study designs may help us understand the relationship of these social, psychological, and demographic factors with postpartum depression among women.

\section{Conclusions}

The current study highlights the substantial burden of postpartum depression among women in Sindh, Pakistan. Low education status, family type, and gender of the newborn did not significantly correlate with postpartum depression. However, exclusive formula milk feeding was associated with an increased incidence of PPD among the study population. Interestingly, almost two-fifths of the participants, fed formula milk to their newborns. In short, there is a dire need for attention from the healthcare providers to cater to the mental health needs of pregnant women in our setups.

\section{Additional Information}

\section{Disclosures}

Human subjects: Consent was obtained by all participants in this study. Jinnah Sindh Medical University issued approval JSMU/IRB/2019/-167. This is to inform you that the current study, entitled, "Postpartum Depression: Prevalence and Associated Risk Factors among Women in Sindh, Pakistan" has been approved by the IRB committee. . Animal subjects: All authors have confirmed that this study did not involve animal subjects or tissue. Conflicts of interest: In compliance with the ICMJE uniform disclosure form, all authors declare the following: Payment/services info: All authors have declared that no financial support was received from any organization for the submitted work. Financial relationships: All authors have declared that they have no financial relationships at present or within the previous three years with any organizations that might have an interest in the submitted work. Other relationships: All authors have declared that there are no other relationships or activities that could appear to have influenced the submitted work.

\section{References}

1. Hadi H, Hadi S: Uncovering the concealed part of motherhood-postpartum depression in mothers . Clin Mother Child Health. 2015, 12:1-4.

2. Rukh R, Kafeel H, Naveed S, Sarwar G: Prevalence of postpartum depression in primigravida and multigravida with normal physiological status. J Pharm Pharmaceut Sci. 2013, 17:16-20.

3. Husain N, Chaudhry N, Furber C, et al.: Group psychological intervention for maternal depression: a nested qualitative study from karachi, pakistan. World J Psychiatr. 2017, 22:98. 10.5498/wjp.v7.i2.98

4. Husain N, Zulqernain F, Carter LA, et al.: Treatment of maternal depression in urban slums of Karachi, Pakistan: a randomized controlled trial (RCT) of an integrated maternal psychological and early child development intervention. Asian J Psychiatr. 2017, 29:63-70. 10.1016/j.ajp.2017.03.010

5. Gulamani SS, Shaikh K, Chagani J: Postpartum depression in Pakistan. Nurs Womens Health. 2013, 17:14752. 10.1111/1751-486X.12024

6. Bhusal BR, Bhandari N, Chapagai M, Gavidia T: Validating the Edinburgh postnatal depression scale as a screening tool for postpartum depression in Kathmandu, Nepal. Int J Ment Health Syst. 2016, 1:71. 10.1186/s13033-016-0102-6

7. O'hara MW, Swain AM: Rates and risk of postpartum depression-a meta-analysis . Int Rev Psychiatry. 1996, 8:37-54. 10.3109/09540269609037816

8. Halbreich U, Karkun S: Cross-cultural and social diversity of prevalence of postpartum depression and depressive symptoms. J Affect Disord. 2006, 91:97-111. 10.1016/j.jad.2005.12.051

9. Clout D, Brown R: Marital relationship and attachment predictors of postpartum stress, anxiety, and depression symptoms. J Consult Clin Psychol. 2016, 35:322-41. 10.1521/jscp.2016.35.4.322

10. Kalar MU, Fatima I, Nabila K, et al.: Prevalence and predictors of postnatal depression in mothers of Karachi. Int J Collab Res Intern Med Public Health. 2012, 4:830-839.

11. Cox JL, Holden JM, Sagovsky R: Detection of postnatal depression: development of the 10-item edinburgh postnatal depression scale.. Br J Psychiatry. 1987, 150:782-6. 10.1192/bjp.150.6.782

12. Hahn-Holbrook J, Cornwell-Hinrichs T, Anaya I: Economic and health predictors of national postpartum depression prevalence: a systematic review, meta-analysis, and meta-regression of 291 studies from 56 countries. Front Psychiatry. 2018, 8:248. 10.3389/fpsyt.2017.00248

13. Upadhyay RP, Chowdhury R, Salehi A, et al.: Postpartum depression in India: a systematic review and metaanalysis. Bull World Health Organ. 2017, 95:706. 10.2471/blt.17.192237

14. Khan MM, Hanan A, Tahir M, Tahir T, Raza SS, Shafique MS.: Frequency of postpartum depression in women attending fauji foundation hospital peshawar. J Med Sci. 2015, 11:190-3.

15. Matsumura K, Hamazaki K, Tsuchida A, Kasamatsu H, Inadera H: Education level and risk of postpartum depression: results from the japan environment and children's study (JECS). BMC psychiatry. 2019, 19:419. 10.1186/s12888-019-2401-3

16. Ystrom E: Breastfeeding cessation and symptoms of anxiety and depression: a longitudinal cohort study . 


\section{Cureus}

BMC Pregnancy Childbirth.. 2012, 12:36. 10.1186/1471-2393-12-36

17. Naveed A, Naz F: Risk factors for postpartum depression, interpersonal relationship anxiety, neuroticism and social support in women with postpartum depression. Pak J Soc Sci. 2015, 35:731-57.

18. Nunes AP, Phipps MG: Postpartum depression in adolescent and adult mothers: comparing prenatal risk factors and predictive models. Matern Child Nutr Health J. 2013, 1:1071-9. 10.1007/s10995-012-1089-5

19. Kim TH, Connolly JA, Tamim H: The effect of social support around pregnancy on postpartum depression among Canadian teen mothers and adult mothers in the maternity experiences survey. BMC Pregnancy Childbirth. 2014, 1:162. 10.1186/1471-2393-14-162 